\title{
Graphene-Enabled Adaptive Infrared Textiles
}

\author{
M. Said Ergoktas, Gokhan Bakan, Pietro Steiner, Cian Bartlam, Yury Malevich, Elif Ozden-Yenigun, \\ Guanliang He, Nazmul Karim, Pietro Cataldi, Mark A. Bissett, Ian A. Kinloch, Kostya S. Novoselov, \\ and Coskun Kocabas*
}

Cite This: https://dx.doi.org/10.1021/acs.nanolett.0c01694

Read Online

ACCESS | 世 Metrics \& More 四 Article Recommendations

Supporting Information

ABSTRACT: Interactive clothing requires sensing and display functionalities to be embedded on textiles. Despite the significant progress of electronic textiles, the integration of optoelectronic materials on fabrics remains as an outstanding challenge. In this Letter, using the electro-optical tunability of graphene, we report adaptive optical textiles with electrically controlled reflectivity and emissivity covering the infrared and near-infrared wavelengths. We achieve electro-optical modulation by reversible intercalation of ions into graphene layers laminated on fabrics. We demonstrate a new class of infrared textile devices including display, yarn, and stretchable devices using natural and synthetic textiles. To show the promise of our approach, we fabricated an active device directly onto a tshirt, which enables long-wavelength infrared communication via modu-

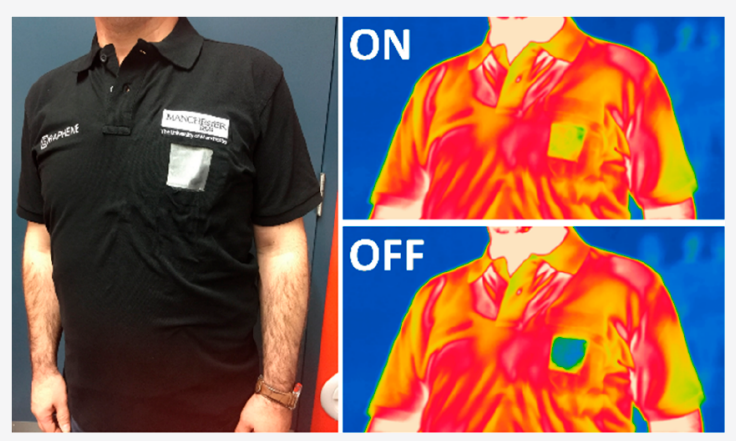
lation of the thermal radiation from the human body. The results presented here provide complementary technologies which could leverage the ubiquitous use of functional textiles.

KEYWORDS: Infrared emissivity, graphene, optoelectronics, thermal camouflage, functional textile

$\mathrm{T}$ he rapid progress of consumer electronics underpins the development of wearable technologies which have already reached a market value worth more than $\$ 50$ billion this year. ${ }^{1}$ Textiles are the natural host material for emerging wearable devices. The key manufacturing challenge for smart wearable textiles is the large-scale integration of electronic/ optical materials into fibers, yarns, and fabrics. Early works on embedding responsive materials such as shape memory alloys, $\mathrm{pH}$, or humidity-sensitive polymers form the foundations of smart textile research. ${ }^{2}$ The integration of devices into fabrics presents a more challenging task as conventional wafer-based microfabrication methods require adaptations into soft and nonplanar forms. ${ }^{3}$ Alternatively, wearable technologies can be developed as stand-alone units such as wearable accessories or stick-on patches on a variety of flexible substrates. ${ }^{4}$ In contrast, integrating technologies directly into/onto clothes necessitates working with traditional textile materials and methods, while taking advantage of the ubiquity of these materials and wellestablished manufacturing infrastructure. Small-scale integrated circuits, ${ }^{5}$ thermal management, ${ }^{6-8}$ therapeutic patches, ${ }^{9}$ sensors, ${ }^{10,11}$ energy harvesting/storage, ${ }^{12}$ and haptic feedback $^{13}$ are among the common smart textile applications.

Integration of optoelectronic devices on textiles deserves a special mention as it promises applications across the electromagnetic spectrum ranging from visible ${ }^{14-16}$ to infrared $^{17-19}$ even further to wireless communication wavelengths. ${ }^{20-22}$ Applications using infrared light are especially appealing as the human body is a near-ideal blackbody infrared source. Emerging technologies such as thermal management and adaptive camouflage require new abilities to regulate the infrared radiation from the surface while keeping the body temperature constant. New textile manufacturing technologies are being explored to achieve such capabilities. For example, Hsu et al. demonstrated enhanced radiative cooling of a human body using nanoporous polyethylene fabric. ${ }^{17}$ Recently, they have also demonstrated a polyethylene textile varying emissivity between $\sim 0.9$ and $\sim 0.3$ when flipped inside out. ${ }^{18}$ In contrast, a dynamic modulation was reported by Zhang et al. using textile consisting of cellulose bimorph fibers and carbon nanotubes. ${ }^{19}$ The infrared transmission is modulated between $\sim 15$ and $\sim 35 \%$ by changing the relative humidity from 5 to $90 \%$. A dynamic modulation of the infrared emissivity between $\sim 0.95$ and $\sim 0.75$ was demonstrated by Mao et al. based on a thermally triggered insulator-to-metal phase transition of $\mathrm{VO}_{2}$ powder integrated into cotton fabric. ${ }^{20}$ The state-of-the-art can be significantly improved by enhancing the modulation range and, more importantly, conceiving a more convenient method for controlling the infrared emission.

Received: April 21, 2020

Revised: June 8, 2020 

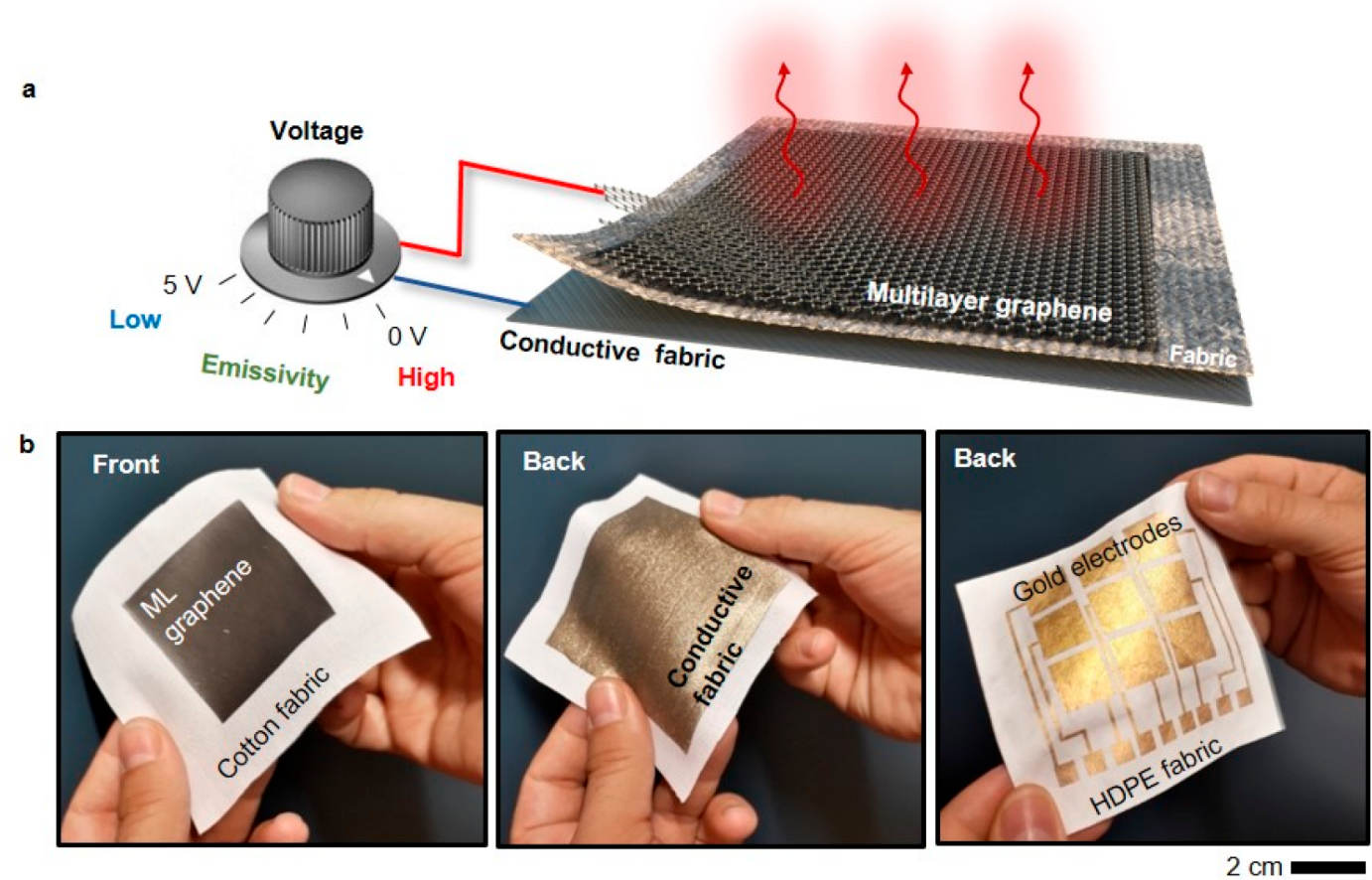

Figure 1. Adaptive infrared textile. (a) Illustration of the textile device with various laminated layers: multilayer graphene, fabric separator, and back electrode layer. The infrared-transparent polymer coating on the graphene layer is not shown for clarity. The infrared emissivity of the device is modulated by applying a voltage difference between the top graphene layer and back electrode layer to initiate intercalation of ions into the graphene layers. (b) Representative examples of fabricated devices on various textile materials such as woven cotton fabric and nonwoven highdensity polyethylene fabric. Continuous conductive textiles or patterned gold electrodes can be used as the back electrode.

Specifically, electro-optical control of the infrared emissivity can provide dynamic, fast, and on-demand modulation that will enable integration with other wearable technologies, such as sensors and integrated circuits. However, this aim could not be achieved so far due to the lack of electro-optical infrared materials and technical challenges associated with the integration schemes.

Graphene and other two-dimensional materials provide new opportunities for functional textiles. ${ }^{23,24}$ The examples in the literature, however, rely on the electrical conductivity of these atomically thin materials. We have been investigating graphene as an adaptive optical platform operating from the visible to microwave wavelengths. ${ }^{25-27}$ In our recent work, we have shown that thermal radiation from multilayer graphene can be modulated electrically via intercalation of ions. ${ }^{28}$ Here, we introduce an optical textile technology by integrating dynamic infrared devices based on electrically tunable, chemical vapor deposition (CVD)-grown graphene into textiles. The materials and the integration scheme reported here are compatible with the state-of-the-art large-area textile processing and a variety of textile materials, including, but not limited to, cotton, polyester, nonwoven synthetics, conductive textiles, and yarns. The reliability and scalability of the developed technology are thoroughly investigated to assess its potential as a wearable functional textile. The versatility of applications is demonstrated by two showcase applications: merging sensing and display capabilities on a multipixel textile device and communicating a message in the long-wavelength infrared (LWIR) by modulating the radiation from the human body.

The devices consist of laminated layers of infraredtransparent polymer layer, CVD-grown multilayer graphene (MLG), a fabric separator layer, and conductive fabric. Figure 1a shows a schematic drawing of a device and its operation. The fabrication starts with growing multilayer graphene layers on Ni foils (detailed in Materials and Methods in Supporting Information). A thin polyethylene (PE) film that functions as an infrared-transparent protective layer is laminated on multilayer graphene prior to etching the $\mathrm{Ni}$ foil. MLG on the polyethylene sheet is attached onto a fabric (e.g., a cotton twill weave), with hot melt adhesive. Good adhesion between MLG and the substrate is extremely important for any wearable application, thus we tested the stability of our MLG on the textile under compression and during washing. To this end, some of the MLG on textile samples was subjected to mechanical compression tests (Figure S1) and washing cycles (Figures S2 and S3). The sheet resistance and infrared emissivity of MLG attached on cotton textile shows no sign of deterioration after the mechanical and washing tests, showing the high endurance and flexibility of the devices. The back electrode (e.g., conductive fabric) is fused to the backside of the fabric, forming the complete device (Figure 1a). In the final step, the ionic liquid electrolyte $\left(\mathrm{BMIMPF}_{6}\right)$ is applied onto the back electrode and allowed to diffuse into the textile substrate. The textile acts as both the separator and ionic conductive layer, allowing ionic motion when a voltage difference is applied to MLG and the back electrode. Figure $1 \mathrm{~b}$ shows representative examples of the fabricated devices on the natural and synthetic textile materials, woven cotton and nonwoven high-density polyethylene clothing, respectively (see Figure S4 for more examples). We tested various back electrode materials including silver-based conductive textiles, stainless steel mesh, sputtered gold, graphene, and reduced graphene oxide. The electrochemical stability of the back electrode plays a critical role in the long-term stability of the 

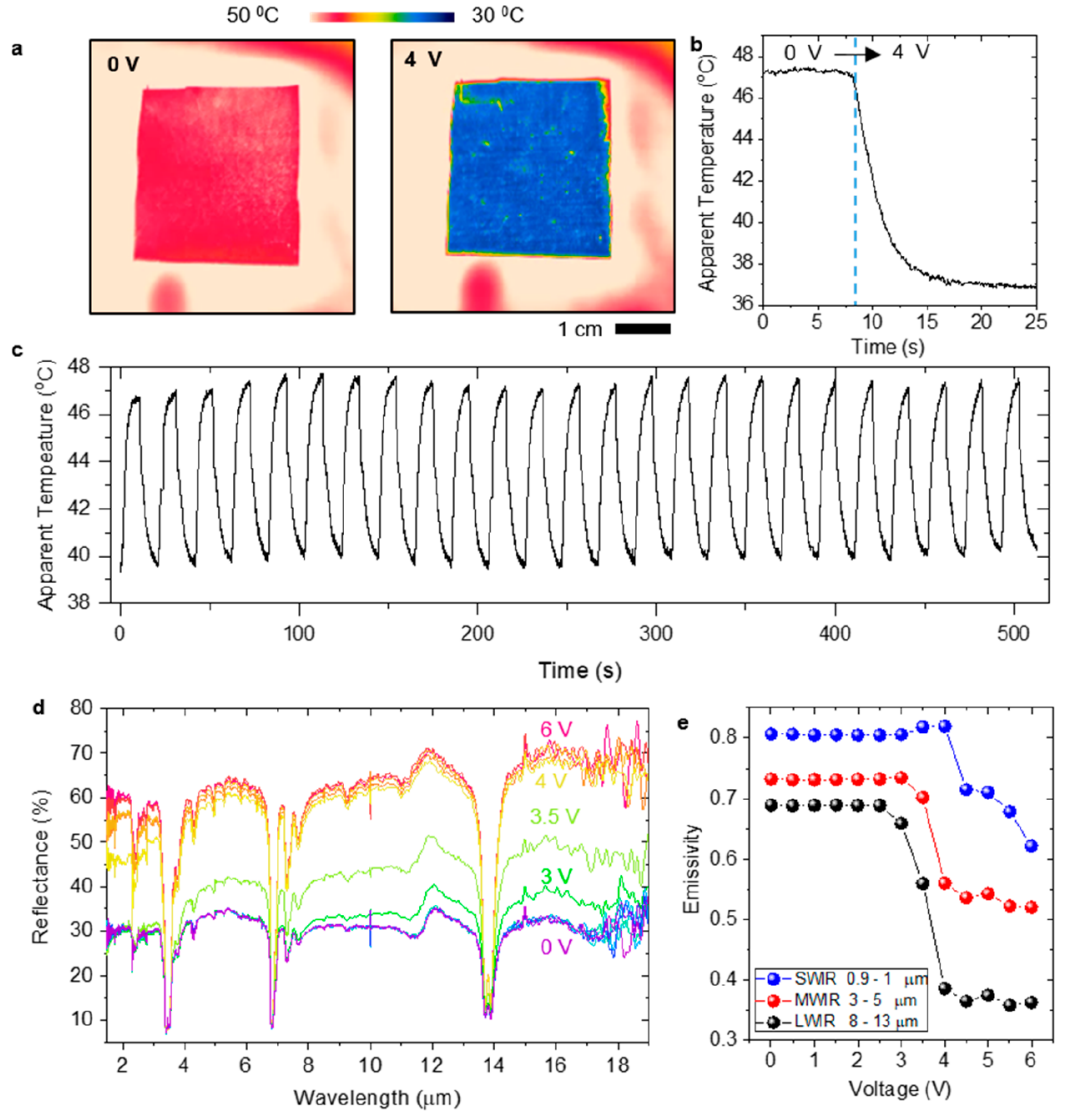

Figure 2. Dynamic control of infrared emission on textile. (a) Thermal images of a cotton-based textile device recorded with a long-wavelength $(8-13 \mu \mathrm{m})$ infrared camera in high emissivity $(0 \mathrm{~V})$ and low emissivity $(4 \mathrm{~V})$ states. The device is placed on a hot plate at $55^{\circ} \mathrm{C}$. (b) Temporal change in the apparent temperature of the device after applying $4 \mathrm{~V}$. (c) Long-term temporal variation in the apparent temperature under a periodic square voltage waveform $(-2$ and $4 \mathrm{~V}$ ) with a period of $20 \mathrm{~s}$. (d) Infrared reflection spectra of the device recorded as the applied voltage varies from 0 to $6 \mathrm{~V}$. (e) Average emissivity of the device in the short-, medium-, and long-wavelength infrared regimes as a function of the applied voltage. The threshold voltage of the device is $2.5 \mathrm{~V}$ for LWIR.

device. An array of patterned back electrodes and wiring on the textile can be fabricated with photolithography followed by metallization and a lift-off process (Figure $1 \mathrm{~b}$ and Materials and Methods in Supporting Information). These pixel electrodes allow us to define dynamic infrared patterns on a continuous graphene layer via area-selective intercalation.

The working principle of the devices is based on reversible intercalation of the ions into the graphene layers and modulating its electrical and optical properties (refer to ref 28 for an insight into the intercalation process). At $0 \mathrm{~V}, \mathrm{MLG}$ has high infrared absorption which leads to high emissivity, revealing the actual temperature of the device (Figure 2a). When a sufficient voltage difference is applied $(>2.5 \mathrm{~V})$, the ionic liquid intercalates into the graphene layers, enhancing the optical conductivity and suppressing the emissivity, therefore concealing the actual device temperature. The thermographs of the device are recorded with a long-wavelength infrared camera which renders images based on the Stefan-Boltzmann law, $P=$ $\varepsilon \sigma T^{4}$, where $P$ is the amount of incident thermal radiation on the bolometer array, $\varepsilon$ is the emissivity of the surface kept constant during rendering, $\sigma$ is the Stefan-Boltzmann constant, and $T$ is the temperature of the surface in Kelvin. The temperature rendered by the camera (apparent temperature, $T_{\mathrm{a}}$ ) is a function of $P$, hence $\varepsilon$ and $T$, and the emissivity setting of the camera, $\varepsilon_{\mathrm{a}}$ (typically 1$): T_{\mathrm{a}}=T\left(\varepsilon / \varepsilon_{\mathrm{a}}\right)^{1 / 4}$. If the background emission reflected from the sample surface is non- negligible, it has to be accounted for. The textile devices are kept in thermal contact with the heat sources, such as the human body, to prevent false screening of the source temperature. Furthermore, the MLG functions as a highly thermally conductive layer that doubles the in-plane thermal diffusivity of the textile, enhancing heat conduction from the source to the surface (Figure S5). The temporal response of the devices was captured by recording thermal videos to obtain the variation in the apparent temperature of the surface (Figure $2 \mathrm{~b}$ and Supporting Video 1). Complete intercalation (suppressing emissivity) takes $\sim 5 \mathrm{~s}$ when the device current is not limited. Note that these measurements were recorded in a closed lab environment which has background thermal radiation $\left(21^{\circ} \mathrm{C}\right)$, limiting the minimum apparent temperature (see Figure S6). The devices can be cycled steadily between high and low emissivity states many times (Figure 2c), however, exceeding the electrochemical window of the electrolyte degrades the device performance.

The emissivity modulation was quantified by infrared and near-infrared reflection measurements using a Fourier transform infrared (FTIR) spectrometer equipped with an integrating sphere. At $0 \mathrm{~V}$, the reflectance of the intrinsic device is almost flat at $\sim 30 \%$ except for the fingerprint absorptions of the top polyethylene film at wavelengths $\sim 3.4$, $\sim 6.8, \sim 13.9 \mu \mathrm{m}$ and atmospheric absorptions (e.g., $\mathrm{CO}_{2}$, $\mathrm{H}_{2} \mathrm{O}$ ) (Figure 2d). In the spectral sensitivity range of the 

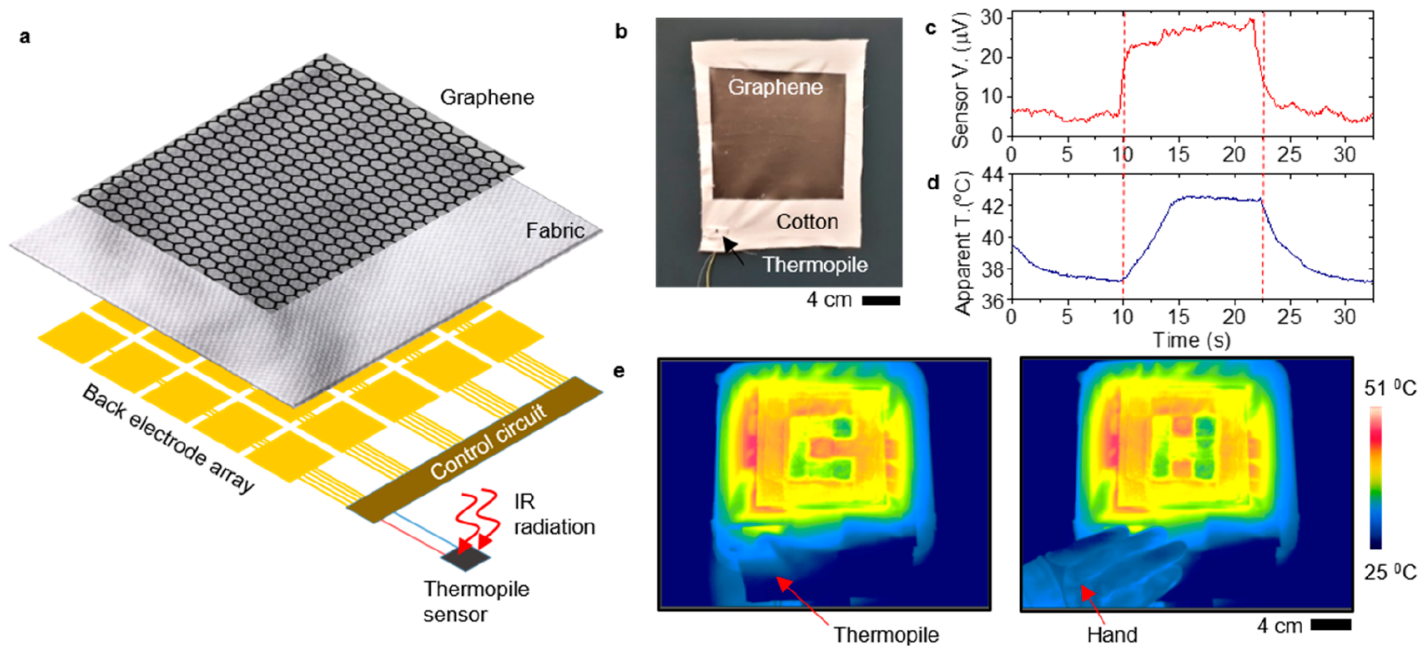

Figure 3. Sensing and display on adaptive textile. (a) Illustration of a textile device with sensing and display capabilities. A large-area graphene (15 $\times 15 \mathrm{~cm}^{2}$ ) layer was laminated onto woven cotton fabric. Individually addressable $5 \times 5$ gold electrode array on the back of the textile was used to control the local emissivity of the device. A thermopile sensor was used to sense the presence of a hot object. An external electronic circuit processed the sensor signal and controlled the voltages of the individual pixels. (b) Photograph of the device with the attached thermopile sensor. (c) Variation of the output voltage of the sensor and (d) apparent temperature of an active pixel as a hot object, author's hand, is brought over the sensor $(t \approx 10 \mathrm{~s})$ and removed $(t \approx 22.5 \mathrm{~s})$. The dashed lines mark the time when the sensor voltage reaches the pixel threshold voltage of $15 \mu \mathrm{V}$. (e) Thermal images of the textile device displaying letters " $\mathrm{C}$ " and " $\mathrm{H}$ " as a response to the absence and presence of the hand, respectively. The temperature of the hot plate is $55{ }^{\circ} \mathrm{C}$.

thermal camera $(8-13 \mu \mathrm{m})$, such absorptions are minimized owing to the careful selection of the top protection film (see Figure S7 for the transmission spectrum of the polyethylene film). The emissivity (or absorptivity) is calculated as $1-R$, where $R$ is the reflectance, as no light passes through the device. As the ions intercalate the graphene layers, the Fermi energy and the optical conductance of MLG increase, enhancing the infrared reflectance. The Pauli blocking of infrared absorption and the increased Drude optical conductivity of graphene are the main factors in the enhanced infrared reflectance. $^{29}$ The reflectance modulation is more pronounced for the longer wavelengths due to Drude-type behavior of free electrons on graphene. The average emissivity of the device in the wavelength range of $8-13 \mu \mathrm{m}$ is high $(\sim 0.7)$ for $0 \mathrm{~V}$ and is maintained up to a threshold voltage $(\sim 2.5 \mathrm{~V})$ followed by a sharp drop to $\sim 0.35$ for $>4 \mathrm{~V}$ (Figure $2 \mathrm{e})$. These results agree well with the thermographs shown in Figure $2 \mathrm{a}$. The emissivity modulation covers both the longwavelength infrared $(8-13 \mu \mathrm{m})$ and medium-wavelength infrared (MWIR, 3-5 $\mu \mathrm{m}$ ) ranges. In the MWIR, however, the polyethylene film exhibits a major absorption due to $\mathrm{C}-\mathrm{H}$ stretching mode that is unaffected with the applied voltage, limiting the emissivity modulation range to $\sim 0.7-0.5$ (Figure 2e). Thus, applications in this wavelength range necessitate reconsideration of the protection layer. Another effect of the polyethylene layer is the enhanced emissivity of the surface owing to thermal extraction by polyethylene whose refractive index is larger than that of air. ${ }^{30}$ We also observed relatively small emissivity modulation (0.2-0.4) in the short-wavelength infrared (SWIR, $0.9-1.7 \mu \mathrm{m}$ ) range. Nonetheless, the modulation in the SWIR can be detected by a silicon CCD camera with a near-infrared cut-on filter (Figure S8). The modulation in the visible spectrum is negligible due to insufficient doping of graphene. The optical modulation can be enhanced in the near-infrared and further pushed toward the visible regime with the use of a textile-compatible ionic liquid with a larger electrochemical window.
The natural deformation and drapeability of a textile in mechanically active environments is accounted for using highly stretchable elastane knitted fabric. Unlike graphene, the CVDgrown polycrystalline MLG is not stretchable due to the defects and grain boundaries. ${ }^{31}$ Therefore, a buckled MLG structure is used on the stretchable textile device to accommodate strain levels up to $60 \%$ (see Supporting Information Note 1 and Figure S9). Integration of graphene on textile is also extended to low-dimensional yarns to take advantage of scaling the technology by interlacing infraredactive yarns (see Supporting Information Note 2, Figure S10, and Figure S11, discussing the results on the yarn devices). To further improve the stability of the devices, fixation methods can be applied on the textile to localize the ionic liquid electrolyte. Polymer matrix such as polyethylene glycol can be used to stabilize the ionic liquid inside the textile.

The electrically controlled emissivity of the textile devices together with the complex electrode patterns and embedded sensors can pave the way for interactive clothing. Such a device can serve multiple functions such as adaptive thermal camouflage or textile display. Figure 3 demonstrates such an example by combining the adaptive infrared response and display functions using a device with an array of 25 individually addressable electrodes and a thermopile sensor (Figure 3a). A large single-piece MLG sheet on cotton fabric was used as the active layer (Figure $3 \mathrm{~b}$ ). Each electrode controls the emissivity of an area of $2 \times 2 \mathrm{~cm}^{2}$. An external electronic circuit was programmed to react to the heat signature on the sensor. The graphs in Figure 3c,d show the sensor signal and apparent temperature of an active pixel. The multipixel textile device shows letters "C" or " $\mathrm{H}$ " (representing cold and hot, respectively) by tuning the emissivity of corresponding pixels interacting to the absence or presence of a hot object over the sensor. Figure $3 \mathrm{e}$ shows the thermal images during the operation of the device as it alternates between the two letters when a hand is placed over the radiation sensor (see Supporting Video 2). The pixel size is limited by the surface 
roughness and the thickness of the textile. We anticipate that the pixels can be scaled down to $100 \mu \mathrm{m}$ on smooth and thin textiles. We also tested graphene-based capacitive touch sensors and strain sensors on textiles, which could provide more complex feedback from the surrounding of the device. The demonstration highlights the capability of creating complex adaptive patterns and sensing capabilities, which would inspire other applications such as adaptively blending the thermal signature of the device into a dynamic background.

In the final part of the paper, to further highlight the promise of this adaptive textile, we demonstrate LWIR communication on a t-shirt by electrically modulating the infrared radiation of the human body. Due to natural body temperature, in ambient conditions, the human body radiates $\sim 100 \mathrm{~W}$ of infrared light mainly in the LWIR range. This spectral range also coincides with the atmospheric transmission window which enables longdistance propagation of the emitted LWIR light. Without using an additional light source, we were able to send messages undetectable by the naked eye or visible cameras by modulating the emissivity of a $\mathrm{t}$-shirt. The $\mathrm{t}$-shirt device was fabricated by laminating a $6 \times 6 \mathrm{~cm}^{2}$ MLG/PE film directly on a $100 \%$ cotton $t$-shirt surface and a stainless steel mesh to the back side (Figure 4a). We used a battery-powered microcontroller (Arduino UNO) to apply an encoded waveform generated by a pulse-width-modulated digital voltage that was programmed to communicate the initial letters of National Graphene Institute, "N", "G", and "I", in Morse code. The dash and dot signals were created by suppressing the apparent temperature for long ( $9 s)$ and short (3s) durations. Figure $4 b, c$ shows the infrared snapshots of the t-shirt at the high and low emissivity states. The graph shows the recorded apparent temperature from a distance of $3 \mathrm{~m}$. The experiment was conducted outdoors to take advantage of the lower background temperature.

This demonstration differs from the LWIR free-space optical communication that mainly aims transmitting data at high speed using high power infrared sources, such as quantum cascade lasers. ${ }^{32}$ The main advantage of this demonstration is the use of the human body as a power source. Another advantage is that it prevents detection of the communicated message by the naked eye or visible cameras. The use of a microcontroller further allows building more sophisticated circuitry on textiles, in turn enabling more secure communication protocols, for instance, initiation of the communication upon receiving an external triggering stimuli. The speed of communication using a single patch is limited by the intercalation/deintercalation process, which scales with the area of the device. Thus, the overall communication speed can be enhanced using multiple smaller patches and parallel processing of the message. Alternatively, a multipixel display, similar to the one employed in Figure 3, can be used to communicate alphanumerical characters or complex patterns. The size of a patch or a pixel depends on the imaging distance and the resolution of the thermal camera. This demonstration emphasizes the natural adaptability of the developed technology to everyday apparel such as a t-shirt in a realistic environment, the use of body temperature for the operation, and the portability of the technology through the use of a small-scale and lightweight controller and power source. Supporting Video 3 shows communication of the full message.

The use of small electrical signals for modulation of the infrared emissivity is a significant advantage over the alternatives as it enables an adaptive response, a necessity for

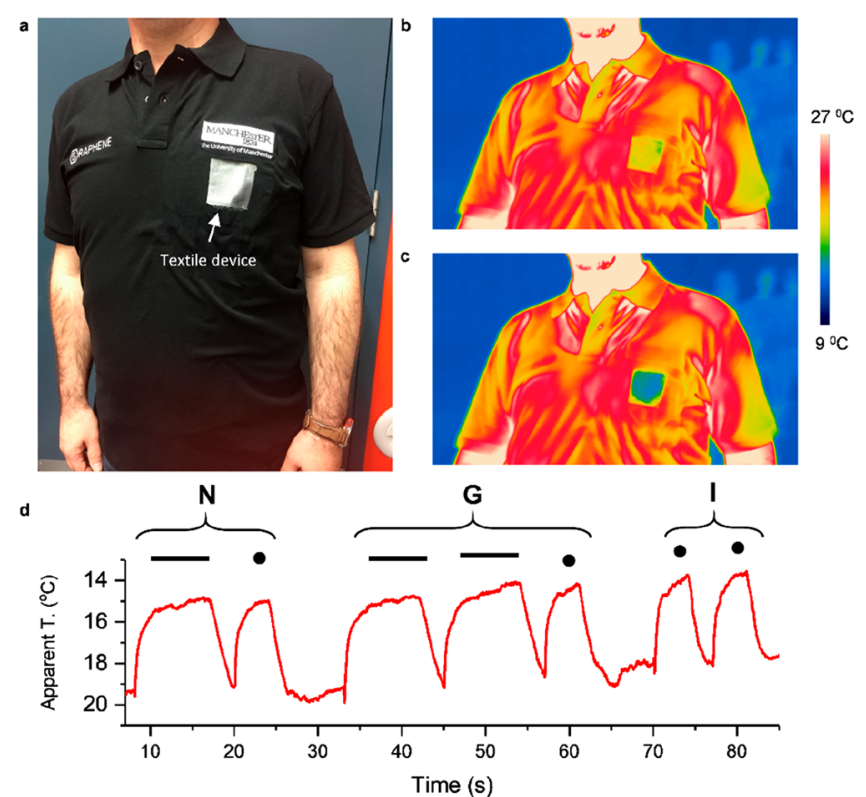

Figure 4. Infrared communication on a t-shirt. (a) Photograph and thermographs for (b) high emissivity and (c) low emissivity states of a device fabricated on a $t$-shirt. The infrared emission from the author's body is modulated to communicate a message in the infrared ( $t$-shirt logo copyright permission from University of Manchester). (d) Modulation of the apparent temperature of the device communicating letters "N", "G", and "I" in Morse code. Temperature scale is reversed as the apparent temperature is suppressed when the device is on.

dynamic thermal camouflage and thermal management applications. The developed technology requires low voltage $(\sim 3 \mathrm{~V})$ and low energy $\left(5.5 \times 10^{-4} \mathrm{mAh} / \mathrm{cm}^{2}\right.$ per intercalation event, corresponding to a charge density of $\sim 10^{14} \mathrm{~cm}^{-2}$ for each graphene layer; see Figure S12). Therefore, a compact coin cell with $1000 \mathrm{mAh}$ capacity can turn on a t-shirt size $(\sim 1$ $\mathrm{m}^{2}$ ) device $\sim 180$ times. Effectively operating as a capacitor, energy is consumed only during the charging (intercalation) cycle. Thus, the average standby power is virtually zero, enabling hours or days of operation depending on the switching frequency. The low voltage and low-power operation highly enhance the portability of the developed technology through the use of a miniature power supply and peripheral circuitry. In contrast, the alternative method (directly heating a surface) to control the thermal emission necessitates $\sim 100 \mathrm{~W}$ for the same area. A coin cell battery can power such a device for only $\sim 100 \mathrm{~s}$. Directly cooling the surface with the help of Peltier coolers is even more energy consuming. ${ }^{33}$

In conclusion, this study provides a framework for grapheneenabled adaptive optical textiles. The developed methods for fabrication and transfer of graphene can easily be integrated into well-established textile infrastructure as the methods are compatible with the textile industry and large-area processing. The successful demonstration of the modulation of optical properties on different forms of textile can leverage the ubiquitous use of fibrous architectures and enable new technologies operating in the infrared and other regions of the electromagnetic spectrum for applications including communication, adaptive space suits, and fashion. 


\section{ASSOCIATED CONTENT}

\section{s) Supporting Information}

The Supporting Information is available free of charge at https://pubs.acs.org/doi/10.1021/acs.nanolett.0c01694.

Materials and methods; details of fabrication and measurement procedures; discussion of stretchable devices; discussion of yarn devices; mechanical test and washing cycle results; back electrode options; thermal diffusivity results; calculation of apparent temperature for varying emissivity; transmission spectrum of polyethylene film; near-infrared measurement results; results on stretchable devices; yarn devices; current-time characteristics of a device; illustration of the fabrication process; uniformity and Raman measurement results; infrared properties of textile materials(PDF)

Supporting Video 1. Dynamic infrared emissivity change on a cotton device (MP4)

Supporting Video 2. Operation of an adaptive infrared textile display (MP4)

Supporting Video 3. Infrared communication of letters "N", "G", and "I" consecutively in Morse code by a cotton device on a t-shirt (MP4)

Supporting Video 4. Increasing reflectance of a polyester device in the near-infrared (MP4)

\section{AUTHOR INFORMATION}

\section{Corresponding Author}

Coskun Kocabas - Department of Materials, National Graphene Institute, and Henry Royce Institute for Advanced Materials, University of Manchester, Manchester M13 9PL, United Kingdom; (1) orcid.org/0000-0003-0831-5552; Email: coskun.kocabas@manchester.ac.uk

\section{Authors}

M. Said Ergoktas - Department of Materials and National Graphene Institute, University of Manchester, Manchester M13 9PL, United Kingdom; (1) orcid.org/0000-0002-8749-8571

Gokhan Bakan - Department of Materials and National Graphene Institute, University of Manchester, Manchester M13 9PL, United Kingdom

Pietro Steiner - Department of Materials and National Graphene Institute, University of Manchester, Manchester M13 9PL, United Kingdom

Cian Bartlam - Department of Materials and National Graphene Institute, University of Manchester, Manchester M13 9PL, United Kingdom

Yury Malevich - Department of Materials and National Graphene Institute, University of Manchester, Manchester M13 9PL, United Kingdom

Elif Ozden-Yenigun - School of Design, Textiles, Royal College of Art, London SW7 2 EU, United Kingdom

Guanliang He - Department of Materials, University of Manchester, Manchester M13 9PL, United Kingdom

Nazmul Karim - Centre for Fine Print Research, University of the West of England, Bristol BS16 1QY, United Kingdom; (1) orcid.org/0000-0002-4426-8995

Pietro Cataldi - Department of Materials and National Graphene Institute, University of Manchester, Manchester M13 9PL, United Kingdom; (1) orcid.org/0000-0001-9468-4009

Mark A. Bissett - Department of Materials, National Graphene Institute, and Henry Royce Institute for Advanced Materials,
University of Manchester, Manchester M13 9PL, United

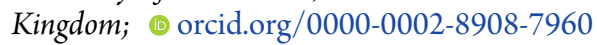

Ian A. Kinloch - Department of Materials, National Graphene Institute, and Henry Royce Institute for Advanced Materials, University of Manchester, Manchester M13 9PL, United Kingdom; 이이.org/0000-0003-3314-6869

Kostya S. Novoselov - National Graphene Institute and Department of Physics and Astronomy, University of Manchester, Manchester M13 9PL, United Kingdom

Complete contact information is available at:

https://pubs.acs.org/10.1021/acs.nanolett.0c01694

\section{Author Contributions}

C.K. conceived the idea. M.S.E. synthesized the graphene samples and fabricated the devices. M.S.E., G.B., and C.K. performed the experiments. G.B., M.S.E., K.S.N., and C.K. analyzed the data and wrote the manuscript. P.S. conducted the thermal diffusivity measurements. E.O.Y. provided the textile samples and consultation on textile integration processes. G.H. and N.K. performed the mechanical tests. P.C. performed the washing tests. C.B., Y.M., M.B., and I.A.K. assisted with manuscript preparation. All authors discussed the results and contributed to the scientific interpretation as well as to the writing of the manuscript.

\section{Notes}

The authors declare the following competing financial interest(s): C.K. is involved activities towards commercialization of variable emissivity surfaces under SmartIR Ltd. The work is subject to patent application by M.S.E., G.B., and C.K.

\section{ACKNOWLEDGMENTS}

This research is supported by European Research Council through ERC-Consolidator grant (Grant No. 682723, SmartGraphene). In addition, we acknowledge Graphene Engineering Innovation Centre (GEIC) for access to the CVD system. C.B. acknowledges EPSRC Doctoral Prize Fellowship EP/R513131/1. E.O.Y. acknowledges the European Union's Horizon 2020 research and innovation programme under Grant Agreement No. 796640. P.C. and I.A.K. acknowledge the European Union's Horizon 2020 research and innovation programme under Grant Agreement No. 785219.

\section{REFERENCES}

(1) Hayward, J. E-Textiles 2019-2029: Technologies, Markets, Players; IDTechEx: Cambridge, MA, 2019.

(2) Mather, R. R. Intelligent Textiles. Rev. Prog. Color. Relat. Top. 2001, 31, 36-41.

(3) Jang, K. I.; Han, S. Y.; Xu, S.; Mathewson, K. E.; Zhang, Y.; Jeong, J. W.; Kim, G. T.; Webb, R. C.; Lee, J. W.; Dawidczyk, T. J.; Kim, R. H.; Song, Y. M.; Yeo, W. H.; Kim, S.; Cheng, H.; Rhee, S. Il; Chung, J.; Kim, B.; Chung, H. U.; Lee, D.; Yang, Y.; Cho, M.; Gaspar, J. G.; Carbonari, R.; Fabiani, M.; Gratton, G.; Huang, Y.; Rogers, J. A. Rugged and Breathable Forms of Stretchable Electronics with Adherent Composite Substrates for Transcutaneous Monitoring. Nat. Commun. 2014, 5, 4779.

(4) Gao, L.; Zhang, Y.; Malyarchuk, V.; Jia, L.; Jang, K. I.; Chad Webb, R.; Fu, H.; Shi, Y.; Zhou, G.; Shi, L.; Shah, D.; Huang, X.; Xu, B.; Yu, C.; Huang, Y.; Rogers, J. A. Epidermal Photonic Devices for Quantitative Imaging of Temperature and Thermal Transport Characteristics of the Skin. Nat. Commun. 2014, 5, 4938.

(5) Yoon, J.; Jeong, Y.; Kim, H.; Yoo, S.; Jung, H. S.; Kim, Y.; Hwang, Y.; Hyun, Y.; Hong, W.-K.; Lee, B. H.; Choa, S.-H.; Ko, H. C. Robust and Stretchable Indium Gallium Zinc Oxide-Based Electronic 
Textiles Formed by Cilia-Assisted Transfer Printing. Nat. Commun. 2016, 7, 11477.

(6) Hong, S.; Gu, Y.; Seo, J. K.; Wang, J.; Liu, P.; Meng, Y. S.; Xu, S.; Chen, R. Wearable Thermoelectrics for Personalized Thermoregulation. Sci. Adv. 2019, 5, No. eaaw0536.

(7) Hsu, P. C.; Liu, X.; Liu, C.; Xie, X.; Lee, H. R.; Welch, A. J.; Zhao, T.; Cui, Y. Personal Thermal Management by Metallic Nanowire-Coated Textile. Nano Lett. 2015, 15 (1), 365-371.

(8) Hu, X.; Tian, M.; Xu, T.; Sun, X.; Sun, B.; Sun, C.; Liu, X.; Zhang, X.; Qu, L. Multiscale Disordered Porous Fibers for SelfSensing and Self-Cooling Integrated Smart Sportswear. ACS Nano 2020, 14 (1), 559-567.

(9) Mostafalu, P.; Kiaee, G.; Giatsidis, G.; Khalilpour, A.; Nabavinia, M.; Dokmeci, M. R.; Sonkusale, S.; Orgill, D. P.; Tamayol, A.; Khademhosseini, A. A Textile Dressing for Temporal and Dosage Controlled Drug Delivery. Adv. Funct. Mater. 2017, 27, 1702399.

(10) Caldara, M.; Colleoni, C.; Guido, E.; Re, V.; Rosace, G. Optical Monitoring of Sweat PH by a Textile Fabric Wearable Sensor Based on Covalently Bonded Litmus-3-Glycidoxypropyltrimethoxysilane Coating. Sens. Actuators, B 2016, 222, 213-220.

(11) Gao, W.; Emaminejad, S.; Nyein, H. Y. Y.; Challa, S.; Chen, K.; Peck, A.; Fahad, H. M.; Ota, H.; Shiraki, H.; Kiriya, D.; Lien, D. H.; Brooks, G. A.; Davis, R. W.; Javey, A. Fully Integrated Wearable Sensor Arrays for Multiplexed in Situ Perspiration Analysis. Nature 2016, 529 (7587), 509-514.

(12) Gong, W.; Hou, C.; Zhou, J.; Guo, Y.; Zhang, W.; Li, Y.; Zhang, Q.; Wang, H. Continuous and Scalable Manufacture of Amphibious Energy Yarns and Textiles. Nat. Commun. 2019, 10, 868.

(13) Yu, X.; Xie, Z.; Yu, Y.; Lee, J.; Vazquez-Guardado, A.; Luan, H.; Ruban, J.; Ning, X.; Akhtar, A.; Li, D.; Ji, B.; Liu, Y.; Sun, R.; Cao, J.; Huo, Q.; Zhong, Y.; Lee, C. M.; Kim, S. Y.; Gutruf, P.; Zhang, C.; Xue, Y.; Guo, Q.; Chempakasseril, A.; Tian, P.; Lu, W.; Jeong, J. Y.; Yu, Y. J.; Cornman, J.; Tan, C. S.; Kim, B. H.; Lee, K. H.; Feng, X.; Huang, Y.; Rogers, J. A. Skin-Integrated Wireless Haptic Interfaces for Virtual and Augmented Reality. Nature 2019, 575 (7783), 473-479.

(14) Rein, M.; Favrod, V. D.; Hou, C.; Khudiyev, T.; Stolyarov, A.; Cox, J.; Chung, C. C.; Chhav, C.; Ellis, M.; Joannopoulos, J.; Fink, Y. Nature 2018, 560, 214-218.

(15) Peng, H.; Sun, X.; Cai, F.; Chen, X.; Zhu, Y.; Liao, G.; Chen, D.; Li, Q.; Lu, Y.; Zhu, Y.; Jia, Q. Electrochromatic Carbon Nanotube/Polydiacetylene Nanocomposite Fibres. Nat. Nanotechnol. 2009, 4 (11), 738-741.

(16) Zhang, Z.; Guo, K.; Li, Y.; Li, X.; Guan, G.; Li, H.; Luo, Y.; Zhao, F.; Zhang, Q.; Wei, B.; Pei, Q.; Peng, H. A Colour-Tunable, Weavable Fibre-Shaped Polymer Light-Emitting Electrochemical Cell. Nat. Photonics 2015, 9 (4), 233-238.

(17) Hsu, P.-C.; Song, A. Y.; Catrysse, P. B.; Liu, C.; Peng, Y.; Xie, J.; Fan, S.; Cui, Y. Radiative Human Body Cooling by Nanoporous Polyethylene Textile. Science 2016, 353 (6303), 1019-1023.

(18) Hsu, P. C.; Liu, C.; Song, A. Y.; Zhang, Z.; Peng, Y.; Xie, J.; Liu, K.; Wu, C. L.; Catrysse, P. B.; Cai, L.; Zhai, S.; Majumdar, A.; Fan, S.; Cui, Y. A Dual-Mode Textile for Human Body Radiative Heating and Cooling. Sci. Adv. 2017, 3 (11), No. e1700895.

(19) Zhang, X. A.; Yu, S.; Xu, B.; Li, M.; Peng, Z.; Wang, Y.; Deng, S.; Wu, X.; Wu, Z.; Ouyang, M.; Wang, Y. H. Dynamic Gating of Infrared Radiation in a Textile. Science (Washington, DC, U. S.) 2019, 363 (6427), 619-623.

(20) Mao, Z.; Wang, W.; Liu, Y.; Zhang, L.; Xu, H.; Zhong, Y. Infrared Stealth Property Based on Semiconductor (M)-to-Metallic (R) Phase Transition Characteristics of W-Doped VO2 Thin Films Coated on Cotton Fabrics. Thin Solid Films 2014, 558, 208-214.

(21) Zhu, S.; Langley, R. Dual-Band Wearable Textile Antenna on an EBG Substrate. IEEE Trans. Antennas Propag. 2009, 57, 926-935.

(22) Roh, J. S.; Chi, Y. S.; Kang, T. J.; Nam, S. W. Electromagnetic Shielding Effectiveness of Multifunctional Metal Composite Fabrics. Text. Res. J. 2008, 78 (9), 825-835.

(23) Abdelkader, A. M.; Karim, N.; Vallés, C.; Afroj, S.; Novoselov, K. S.; Yeates, S. G. Ultraflexible and Robust Graphene Super- capacitors Printed on Textiles for Wearable Electronics Applications. 2D Mater. 2017, 4, 035016.

(24) Sun, F.; Tian, M.; Sun, X.; Xu, T.; Liu, X.; Zhu, S.; Zhang, X.; $\mathrm{Qu}, \mathrm{L}$. Stretchable Conductive Fibers of Ultrahigh Tensile Strain and Stable Conductance Enabled by a Worm-Shaped Graphene Microlayer. Nano Lett. 2019, 19 (9), 6592-6599.

(25) Polat, E. O.; Uzlu, H. B.; Balci, O.; Kakenov, N.; Kovalska, E.; Kocabas, C. Graphene-Enabled Optoelectronics on Paper. ACS Photonics 2016, 3 (6), 964-971.

(26) Kakenov, N.; Balci, O.; Takan, T.; Ozkan, V. A.; Altan, H.; Kocabas, C. Observation of Gate-Tunable Coherent Perfect Absorption of Terahertz Radiation in Graphene. ACS Photonics 2016, 3 (9), 1531-1535.

(27) Balci, O.; Polat, E. O.; Kakenov, N.; Kocabas, C. GrapheneEnabled Electrically Switchable Radar-Absorbing Surfaces. Nat. Commun. 2015, 6 (1), 6628.

(28) Salihoglu, O.; Uzlu, H. B.; Yakar, O.; Aas, S.; Balci, O.; Kakenov, N.; Balci, S.; Olcum, S.; Süzer, S.; Kocabas, C. GrapheneBased Adaptive Thermal Camouflage. Nano Lett. 2018, 18 (7), 45414548.

(29) Mak, K. F.; Ju, L.; Wang, F.; Heinz, T. F. Optical Spectroscopy of Graphene: From the Far Infrared to the Ultraviolet. Solid State Commun. 2012, 152 (15), 1341-1349.

(30) Yu, Z.; Sergeant, N. P.; Skauli, T.; Zhang, G.; Wang, H.; Fan, S. Enhancing Far-Field Thermal Emission with Thermal Extraction. Nat. Commun. 2013, 4, 1730.

(31) Young, R. J.; Kinloch, I. A.; Gong, L.; Novoselov, K. S. The Mechanics of Graphene Nanocomposites: A Review. Composites Science and Technology. Compos. Sci. Technol. 2012, 72, 1459-1476.

(32) Pavelchek, A.; Trissel, R. G.; Plante, J.; Umbrasas, S. LongWave Infrared (10-Micron) Free-Space Optical Communication System. In Free-Space Laser Communication and Active Laser Illumination III; Voelz, D. G., Ricklin, J. C., Eds.; SPIE, 2004; Vol. 5160, pp 247-252.

(33) Schwarz, A. Adaptive Camouflage in the VIS and IR Spectral Range: Main Principles and Mechanisms. In Target and Background Signatures; Stein, K. U., Schleijpen, R. H. M. A., Eds.; SPIE, 2015; Vol. 9653, pp 52-61. 